\title{
Chitosan Copolymer with Activated Carbon as Nanocomposite Through DFT Modeling
}

\author{
Juan Horacio Pacheco-Sánchez* \\ División de Estudios de Posgrado e Investigación del TecNM/Instituto Tecnológico de Toluca, México.
}

*Corresponding author: Juan Horacio Pacheco-Sánchez, División de Estudios de Posgrado e Investigación del TecNM/Instituto Tecnológico de Toluca, México.

To Cite This Article: Juan Horacio Pacheco-Sánchez. Chitosan Copolymer with Activated Carbon as Nanocomposite Through DFT Modeling. Am J Biomed Sci \& Res. 2019 - 4(6). AJBSR.MS.ID.000843. DOI: 10.34297/AJBSR.2019.04.000843

Received: 眥 August 20, 2019; Published: 眥August 26, 2019

\begin{abstract}
Our aim is to simulate nanocomposites through intermolecular interactions among copolymer chitosan (Ch) and activated carbon (AC) in the simplest form of them. Structural properties as pore size distribution are to be investigated in this way. A geometry optimization (DMol3DFT-GGA-PW91) among $\mathrm{C}_{17}$ carbon-ring and a chitosan copolymer unit provides one nanocomposite system. First, we draw sketches of chitosan copolymer and arbitrary carbon rings to determine their structure stabilities through the minimum energy criterion, which is also applied on AC+Ch system. Second, we consider $\mathrm{C}_{\mathrm{n}}$ carbon rings as activated carbon by knowing that average pore size distribution is around ( $9 \AA \AA$ ) according to BET measurements. Some carbon rings known as carbyne, alternate single and triple bonds, and those known as cumulene only have double bonds. The alternation of bond lengths in carbon rings is at least one signal of the consequences of Jahn-Teller effect. Third, we propose pore size distributions of the nanocomposite by means of radial distribution functions. Nanocomposite $\mathrm{AC}+\mathrm{Ch}$ molecular complex system is expected to be applied in medicine as mitigating intoxication in human beings.
\end{abstract}

\section{Introduction}

With the aim to figure out a molecular complex formed through the interaction between a copolymer unit of chitosan and an activated carbon system arranged in planar ring way, adsorption energy has been calculated by means of minimum energy calculations obtained through $\mathrm{DMol}^{3}$ geometry optimization. Chitin is the second most abundant biopolymer in nature. When the degree of deacetylation of chitin reaches about $50 \%$ (depending on the origin of the polymer), it becomes soluble in aqueous acidic media and is called chitosan [1]. Chitosan is applied to remediation of heavy metals in drinking water and other contaminants by adsorption, because chitosan is macropore size [2] for long linear chains sets forming aggregates. In industrial applications, several solids having pores close to molecular dimensions (micropores $<20 \AA$ ) are used as selective adsorbents because of the physicochemical specificity they display towards certain molecules in contrast to the mesoporous substrates (20-500 ̊) and macropores (> 500 Å) [3]. Adsorbents with these selective properties include activated carbon among others [4]. According to the micropore size distributions (MPSD), the most populated results for measurements of pore size of activated carbon is located around $9 \AA$, at the interval 6 - 18 angstroms in the pore size distribution [5].

There is a great variety of applications of activated carbon as an adsorbent material, and it has been used in areas related to the energy, and the environment, generating materials with a high-en- ergy storage capacity [6]. Matrix isolation measurements [7] exhibit that carbon vapor at $3000 \mathrm{~K}$ consists of atomic and molecular carbon of the approximate composition C (20\%), C (10\%), and linear $\mathrm{C}_{3}(70 \%)$; the abundance of larger species is rather low $(<1 \%)$ [8]. Wakabayashi and Krätschmer remark that all odd chains have a closed-shell electronic structure in their ground state (i.e. are singlet states) and that all even chains have just two electrons in a $\pi$ orbital which can host four electrons (i.e. possess a triplet ground state). When copper acetylide or dicopper acetylide are hydrolyzed with hydrochloric acid, they release polyenes in an extracting hydrocarbon medium and a carbonaceous residue containing carbinoid structures [9]. Structures of acetylenic-ring (carbinoid), and cumulenic-rings have been obtained through Montecarlo [10] and DFT geometry optimization [11]. According to Remya (2016) and references there in, properties of planar monocyclic carbon rings have been extensively studied both theoretically and experimentally.

These properties vary with the number $\mathrm{n}$ of carbon atoms, depending on whether $n$ is odd, $n=4 N$, or $n=4 N+2$ where $N$ is an integer. Linear carbons alternating single and triple bonds (-C $\equiv \mathrm{C}-)$ $n$, are known as carbyne or acetylenic structures, while when double bonds are present $(=\mathrm{C}=\mathrm{C}=)_{\mathrm{n}}$ (Liu 2013) the structure is known as cumulene. Polyyne is known as an allotrope carbon having H($\mathrm{C} \equiv \mathrm{C}-\mathrm{J}_{\mathrm{n}} \mathrm{H}$ chemical structure repeating chain, with alternating single and triple bonds [12] and hydrogen at every extremity, corre- 
sponding to hydrogenated linear carbon chain as any member of the polyyne family $\mathrm{HC}_{2 \mathrm{n}} \mathrm{H}$ [13] with sp hybridization atoms. It is known that polyyne, carbyne, carbynoid, cumulene and cumulenic have been actually synthesized as documented by Cataldo [14]. Bond length alternation (BLA) of carbyne pattern is retained in the rings having an even number of atoms [15]. Additional care must be taken with carbyne rings since the Jahn-Teller distortion (the counterpart of Peierls instability in non-linear molecules) is different in the $\mathrm{C}_{4 N}$ and $\mathrm{C}_{4 N+2}$ families of rings $[10,16,17]$

Particularly, nanostructures of planar monocyclic carbon rings $\mathrm{C}_{n}$ with $n=4 N+2$, where $N$ is a natural number, are closed-shell molecules revealing an intriguing competition between conjugated aromaticity, second-order Jahn-Teller [18] and, at large sizes, Peierls instability effects. Consequently, this leads to different stabilization mechanisms that tend to favor one of three following structures: i) a cumulenic ring with full Dnh symmetry, with all bond angles and bond lengths equal; or either of two distorted acetylenic ring structures, of lower $\mathrm{D}(n / 2) h$ symmetry, with alternating ii) bond angles or iii) bond lengths (Torelli 2000). Methods of synthesis of large monocyclic ring structures $(n<30)$ [19] and of experimentally isolating specific size clusters [20] provide relatively precise spectral information for neutral carbon rings as a function of size [21]. These results suggest that aromatic behavior (no bond alternation for $n=4 N+2$ rings, where $N$ is a natural number) is retained for molecules larger than previously predicted by Hartree-Fock [22] (HF) and accepted in structural chemistry.

Intermolecular carbon-carbon interactions and stability have been investigated on $\mathrm{C}_{4 \mathrm{~N}}$ and $\mathrm{C}_{4 \mathrm{~N}+2}$, carbon ring systems for $\mathrm{N}=1$, ... , 7 except $\mathrm{C}_{4 \mathrm{~N}}$ when $\mathrm{N}=1$ using DFT-mGGA-M-06l level of theory with the basis set $6-311++g(d, p)[11]$. The properties of these rings vary with the number $n$ of carbon atoms, depending on whether $n$ is odd, $n=4 N$ or $n=4 N+2$. The $C_{4 N+2}$ rings show cumulenic structures with all the bond lengths being equal, while the $\mathrm{C}_{4 \mathrm{~N}}$ rings possess carbyne type structures with clear bond length alternation. In principle, electronic structure of diatomic molecules has been built through the overlapping knowledge of the interacting atomic orbitals [23]. In this case, the orbitals correspond to bonding $\left(\sigma_{g}, \pi_{g}\right)$ and antibonding $\left(\boldsymbol{\sigma}_{u^{\prime}}, \pi_{\mathrm{u}}\right)$ orbitals of hydrogen, carbon, nitrogen and oxygen diatomic molecules, whose $\mathrm{H}_{2}, \mathrm{C}_{2}, \mathrm{~N}_{2}$, and $\mathrm{O}_{2}$ ground-state electronic configurations are $1 \sigma_{g}^{2}, 1 \sigma_{g}^{2} 1 \sigma_{u}^{2} 1 \Pi_{u}^{4}, 1 \sigma_{g}^{2} 1 \sigma_{u}^{2} 1 \Pi_{u}^{4} 2 \sigma_{g}^{2}$ and $1 \sigma_{g}^{2} 1 \sigma_{u}^{2} 2 \sigma_{g}^{2} 1 \Pi_{u}^{4} 1 \Pi_{g}^{2}$ with $2,8,10$ and 12 valence electrons, respectively. Actually, the reactivity sites in a molecule correspond to the highest occupied molecular orbitals (HOMO) and lowest unoccupied molecular orbitals (LUMO). HOMO as base (donor), and LUMO as acid (acceptor) are particularly important MOs to predict reactivity in many types of reaction $[24,25]$.

In interaction studies of graphene-chitosan [26,27], one monomer of chitosan (MCh) unit stays almost perpendicularly adsorbed on a $\mathrm{C}_{54} \mathrm{H}_{18}$ graphene (G) sheet in T-shape form. DFT-mGGA-M06L was applied to deal with the exchange-correlation energies using all-electron basis set with triple polarization. The minimum energy criterion was applied for determining the structure stability on the $\mathrm{G}+\mathrm{MCh}$ system using seven different geometries; in addition, they checked with the non-complex vibration frequency. On the other hand, studies of adsorption of chitosan on $(5,5)$ and $(5,0)$ BN nanotubes also exhibit chitosan perpendicularly adsorbed to the latter [28]. In this case they applied DFT-LDA, and the exchange-correlation term was modeled with the Perdew-Wang parameterization. The structural stability was based on finding the minimum energy and non-complex vibrational frequencies. Different sites, charge (neutral, cation and anion) and orientations of chitosan, using the monomer unit, were considered. For the $(5,5)$ nanotube, the minimum energy site corresponds to that one when the monomer (for all charges assigned) is perpendicular to the nanotube; only structural stability was found in the anion assignment for the $(5,0)$ nanotube.

Structures of Acetylenic- and cumulenic- rings are obtained from irregular carbon rings. We consider these structures as carbon physically activated, due to the pore size distribution, and since no activating chemical agent has been applied. Activated carbon and chitosan have been independently applied as adsorption materials to increase environmental quality starndards. Then, we expect AC-Ch nanocomposite to have a powerful adsorption property of pollutants that can be applied not only in wastewater treatment, but also in medicine against intoxication, according to the pore size distribution generated on this new material.

\section{Methodology}

The interaction between an activated carbon system (AC) and a unit of chitosan copolymer $(\mathrm{Ch})$ is studied by means of BIOVIA Materials Studio software DFT-DMol ${ }^{3}[29,30]$. The AC system is cumulenic- or acetylenic- ring of double bonds or single-triple bonds, respectively. An even $C_{n}$ carbon atoms number includes $n=4 N+2$ for $\mathrm{N}=1,2,3 \ldots$, which has been considered cumulenic-ring; however, it is acetylenic-ring when the symmetry is $D_{(n / 2) h}[10]$. To obtain the minimum energy each ring is previously optimized by using DFT and converges into a planar molecular carbon ring structure when the symmetry is $\mathrm{n}=4 \mathrm{~N}$ or $\mathrm{N}=4 \mathrm{~N}+2$. The reactants are $\mathrm{AC}+\mathrm{Ch}$, as an example: $\mathrm{C}_{17}+\mathrm{C}_{14} \mathrm{H}_{24} \mathrm{~N}_{2} \mathrm{O}_{9}$, with $\mathrm{C}_{31} \mathrm{H}_{24} \mathrm{~N}_{2} \mathrm{O}_{9}$ product as a new nanocomposite material. All electron calculations to find interaction energies among reactants $\mathrm{AC}+\mathrm{Ch}$ (Activated Carbon + Chitosan) on $\mathrm{DMol}^{3}$ with GGA-PW91 functional [31-35], unrestricted spin, and dnd bases have been accomplished. Connectivity calculations [36] in an few cases where the bond remained very long after geometry optimization were accomplished in our nanocomposite mostly composed of carbon for bond length tolerances from 0.6 to $1.15 \AA$, according to $\mathrm{DMol}^{3}$ on no-bonding to s- and f-shell scheme bond type, and converting representation to Kekulé.

Radial Distribution Functions (RDF - BIOVIA Materials Studio) were calculated after building a periodic cell (Amorphous Cell - BIOVIA Materials Studio) using $1 \mathrm{gr} / \mathrm{cc}$ as density for one unit at room temperature, with universal forcefield to observe the distribution 
of atom distances. RDF is in here considered as something near to the pore size distribution in some manner. Origin software is used for linear regressions of nine-degree polynomial fitting connected by a B-Spline. These calculations have been achieved on RDFs of one thousand points. Energy calculated by means of geometry optimization on each molecular system allows to obtain adsorption energy through the equation:

$$
E_{a d(c h A C)}=E_{(c h+A C)}-E_{(c h)}-E_{(A C)}(1)
$$

\section{Results}

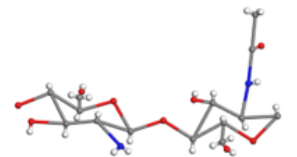

a) Input

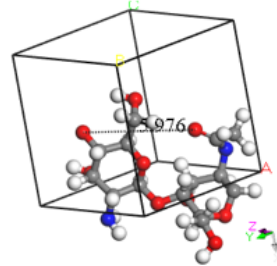

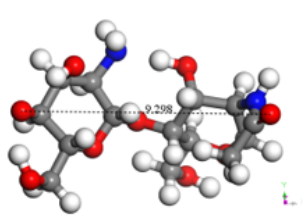

b) output

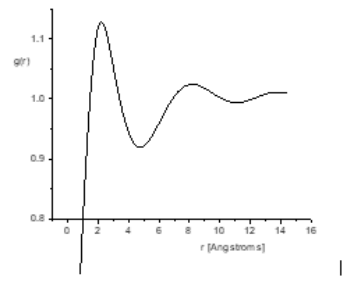

Figure1: Geometry optimization applied to Chitosan copolymer with molecular structure $\mathrm{C}_{14} \mathrm{H}_{24} \mathrm{~N}_{2} \mathrm{O}_{9}$ : a) Input (drawing the chitosan system). b) Output (optimized geometry of this system). c) Periodic cell for this system. d) Radial distribution system in this case. Atoms of: Hydrogen in white color, Nitrogen in blue color, Oxygen in red color and Carbon in gray color.

An input-output chitosan copolymer is observed in Figure 1. The output is a molecular structure resulting after calculating the minimum energy $E_{(c h)}=-5114.7 \mathrm{kcal} / \mathrm{mol}$ through $\mathrm{DMol}^{3}$ DFT-GGAdnd geometry optimization. Considering the whole surface area of the latter molecular unit in Figure 1b, an average micropore size diameter of $9.298 \AA$ is estimated in agreement with micropore size distributions [37]. This diameter estimation also agrees with the corresponding radial distribution function, because each maximum is located as a distribution of atom distances, and we clearly observe two maximums, one at $2.20 \AA$ and another at $8.18 \AA$; however, there is another small maximum around $13.7 \AA$. The first maximum is an average distance very close to the nearest atom neighbours, which is near to the atom bond lenght, the second one provides an average distance from those atoms not so near not so far, and the third maximum give information about the average distance of the far away atoms, the rest atoms do not feel the presence of each other.

The $\mathrm{C}_{17}$ cumulenic-ring double bond among carbons is obtained as shown in Figure 2 by applying DFT geometry optimization and bond type to an irregular system of seventeen single bond carbons, the optimized energy obtained is $E_{\left(c_{17}\right)}=-2653.01 \mathrm{kcal} / \mathrm{mol}$. $\mathrm{D}_{17 \mathrm{~h}} \mathrm{sym}-$ metry on an odd carbon number for monocycle $\mathrm{C}_{17}$ carbon-ring molecular structure is shown in Figure 2a, when alternating long/ short bond lengths are present, as a clear evidence of Jahn-Teller effect due to there is no perfect symmetry. In a perfect symmetry there is equal bond lengths and angles as it is observed in Figure $2 \mathrm{~b}$. In the previous cases when $\mathrm{n}$ is even carbon number the $\mathrm{D}_{\mathrm{nh}}$ symmetry is circular while $\mathrm{D}_{(\mathrm{n} / 2) \mathrm{h}}$ symmetry is a polyhedron. When $\mathrm{n}$ is odd $\mathrm{D}_{\text {nh }}$ exhibits two cases: i) alternating bond lengths showing Jahn Teller effect, and ii) equal bond lengths and angles. The RDF was obtained after building a periodic cell for $\mathrm{C}_{17}$-cumulenic-ring as seen in Figure 2c. Peaks of the radial distribution function are located at $1.89 \AA, 6.26 \AA$, and $12.36 \AA$, according to Figure $2 \mathrm{~d}$.

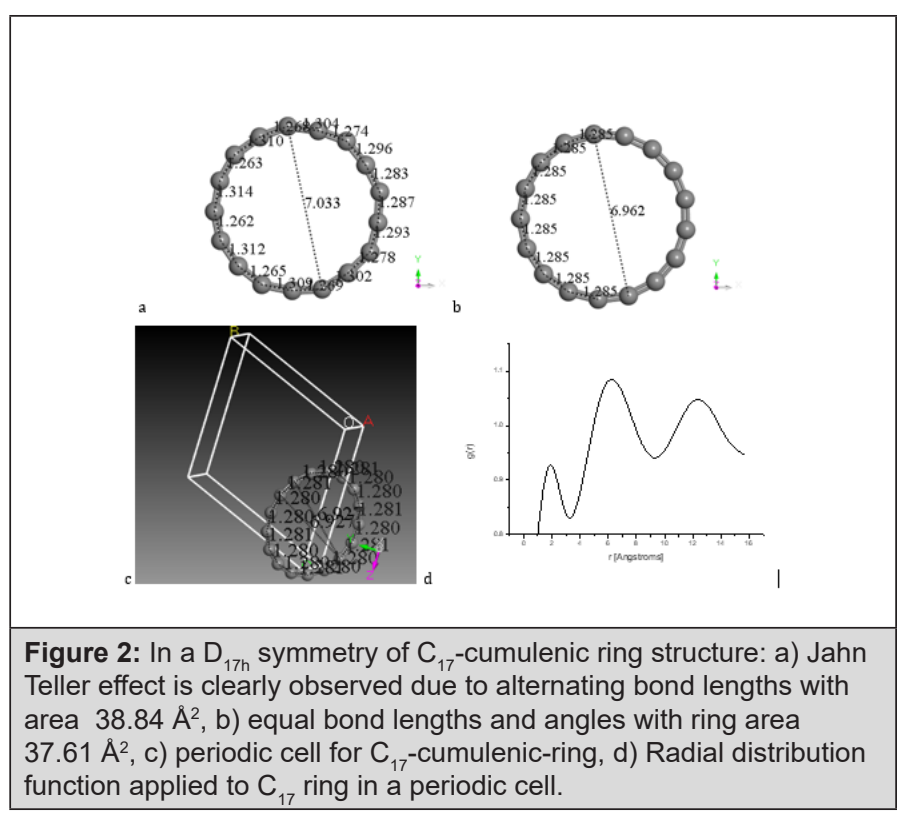

In case of Figure 3, we consider input-output geometry optimization when we join $\mathrm{C}_{17}$-cumulenic ring with chitosan $\mathrm{C}_{14} \mathrm{H}_{24} \mathrm{~N}_{2} \mathrm{O}_{9}$ as in Figure 3a-b, and each one previously at its minimum energy. Then, we applied geometry optimization to the whole system obtaining $E_{\left(c h C_{17}\right)}=-6811.5 \mathrm{kcal} / \mathrm{mol}$. We also considered bond type cri- 
teria and Kekulé representation as shown in Figure 3c. This result is a good example of the possibility of modifying the pore size distribution of chitosan when it is embedded into activated carbon. In order to have a normalized RDF, the nanocomposite is introduced in a periodic cell shown in Figure $3 \mathrm{~d}$, and the radial distribution function is shown in Figure 3e where peaks are located at $2.09 \AA$, $8.09 \AA$, and $13.70 \AA$ A. The product in the output (Figure 3c) is the nanocomposite $\mathrm{C}_{31} \mathrm{H}_{24} \mathrm{~N}_{2} \mathrm{O}_{9}$, and we also applied bond type criteria to observe a change of double bonds to single and triple bonds. The adsorption energy in this case is $E_{(a d)}=-6811.5+5114.7+2653.01=956.3 \mathrm{kcal} / \mathrm{mol}$ according to Equation 1, which corresponds to chemisorption energy agreeing to the range handled by Atkins $[38,39]$.

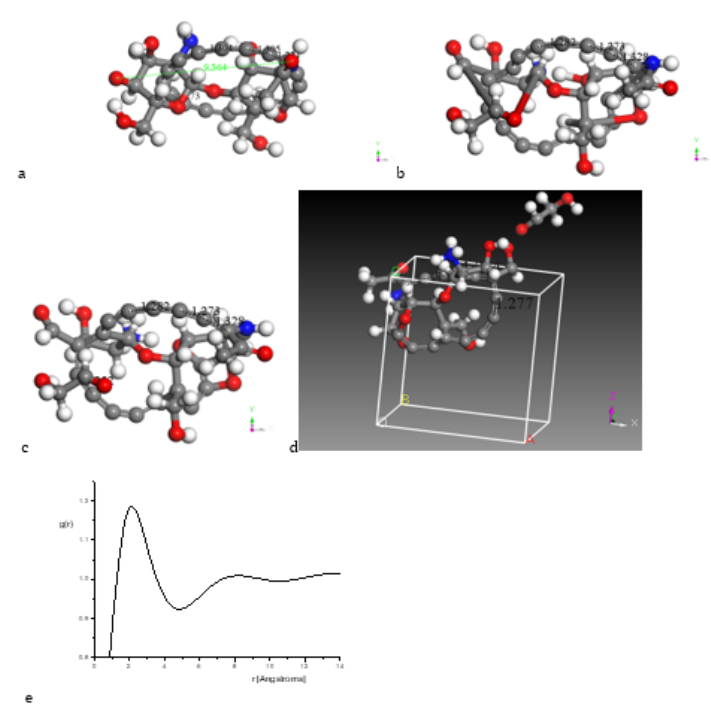

Figure 3: Here we consider input-output geometry optimization: a) Input of a $\mathrm{C}_{17}$-cumulene ring surrounding a chitosan copolymer molecule. b) Output of the previous CA-Chitosan, now as nanocomposite. c) Connectivity criteria applied to nanocomposite. d) This nanocomposite into periodic boundary conditions. e) The radial distribution functions.

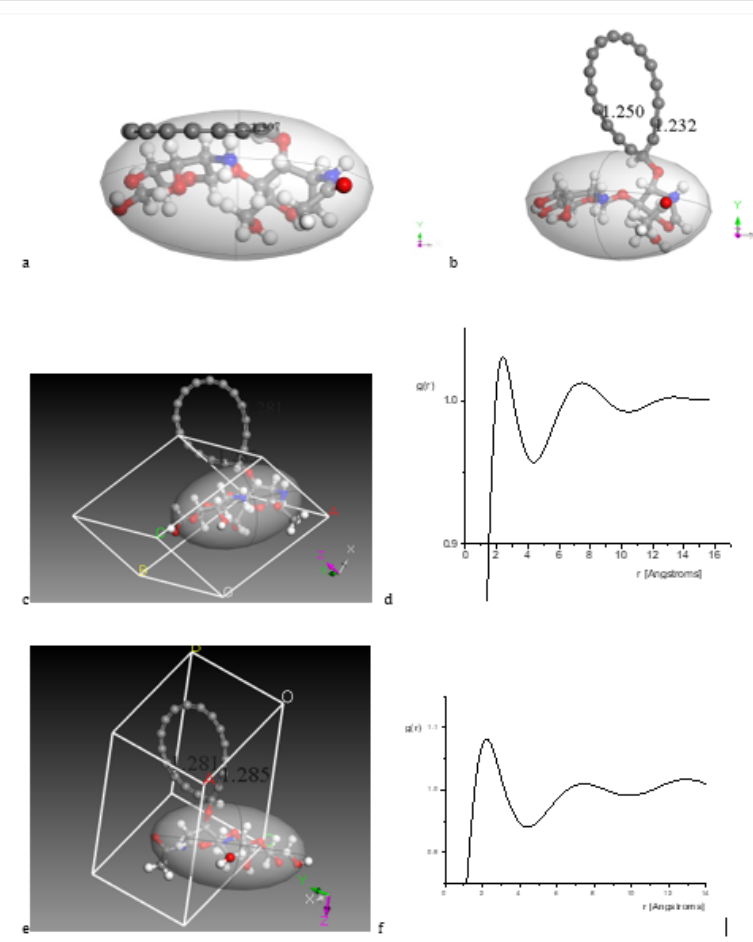

Figure 4: Here we consider another input-output geometry optimization of one $\mathrm{C}_{17}$-cumulenic ring with one chitosan copolymer molecule: a) Input of the reactants in face to face orientation, b) Output of the product in T-shape orientation, now as nanocomposite, c) $\mathrm{C}_{17}$-cumulenic-ring outside the box of periodic boundary conditions, and d) its corresponding RDF, e) $\mathrm{C}_{17}$-cumulenic-ring inside the box, and f) its corresponding RDF.

The $\mathrm{C}_{17}$-cumulenic ring structure surrounding a chitosan copolymer molecule has been optimized in three dimensions using $\mathrm{DMol}^{3}$, in which we clearly observe parts of curved carbon chains passing from double bonds to single and triple bonds. This new nanocomposite has a pore size distribution different to those of the previous $\mathrm{C}_{17}$-cumulenic ring structure alone, or the chitosan 
$\mathrm{C}_{14} \mathrm{H}_{24} \mathrm{~N}_{2} \mathrm{O}_{9}$ copolymer molecule. We considered another configuration moving $\mathrm{C}_{17}$-cumulenic ring in Figure 3a towards face to face orientation as in Figure 4a. We applied minimum energy using $\mathrm{DMol}^{3}$ geometry optimization and the result is shown in Figure $4 \mathrm{~b}$. The $\mathrm{C}_{17}$-cumulenic ring started parallel to the chitosan copolymer and ended perpendicular to it. According to the initial orientation, the T-shape obtained in the nanocomposite product is due to a repulsion on cumulenic carbon atoms in the ring applied by chitosan copolymer, while one carbon of this $\mathrm{C}_{17}$-cumulenic ring inserts one $\mathrm{OH}$ bond of Chitosan copolymer. The optimized energy obtained is $E_{\left(c h+c_{g}\right)}=-7786.709 \mathrm{kcal} / \mathrm{mol}$. In this case, two periodic cells showing different geometry orientations were built, see Figures $4 \mathrm{c}$ and $4 \mathrm{e}$. Peaks of the radial distribution function in Figure 4d are located at $2.37 \AA$, $7.44 \AA$, and $13.04 \AA$; while in Figure 4f are at $2.21 \AA, 7.31 \AA$, and $12.87 \AA$. The adsorption energy in this case is $E_{(a d)}=-7786.709+5114.7+2653.01={ }_{-} 18.99 \mathrm{kcal} / \mathrm{mol}$. The absolute value of this result corresponds to the bond strength of a well of potential, which is an almost physisorption or a very weak chemisorption. In this case the separation of the cumulenic-ring from chitosan copolymer is easier than in the previous case. Then, it is considered that the properties of the reactants are still almost invariants.

\section{Discussion}

Different elongation among single and triple carbon bond lengths in carbynoid-rings, or double bonds in cumulenic-rings, are due to Jahn-Teller effect (Bylaska 1998). Then, when $C_{17}$ cumulenic-ring is optimized, the alternating angles or bond lengths are due to quantum distortion effect. The Jahn-Teller effect is also present in $\mathrm{C}_{12}$ carbynoid-ring, or in $\mathrm{C}_{4 \mathrm{~N}}$ carbynoid-rings, generally. Carbon rings $\mathrm{C}_{4 \mathrm{~N}}(\mathrm{~N}<\sim 8)$ exhibit a substantial first order Jahn-Teller distortion that leads to long/short (single/triple) bond alternation decreasing with increasing $\mathrm{N}$ [16]. The carbon model chosen is among allotropes, from which the most known are fullerenes, nanotubes and graphite; less known is zeolite templated carbon as an example. Polyyne and cumulene are more allotrope forms not so known among many investigators in research community, because they still think these are hypothetical; however, synthesis of them have been accomplished. Again, after practicing connectivity [36], bond type, and Kekulé representation, we infer that bonding type change produced the differences, and the correct values correspond to the correct bonding type in the new molecular complex system formed.

We consider each carbon ring as physically activated through geometry optimization, due to pore size diameter remains in the average size compared against experimental measurements [40]. The optimized $\mathrm{C}_{17}$ carbon-ring system and one optimized chitosan copolymer unit have been studied by considering the result after the corresponding geometry optimization, as a molecular complex obtained when they were joined to form what is also nanocomposite called. In a previous work [41,42], an almost T-shape form between one chitosan copolymer unit and one $\mathrm{C}_{9}$-cumulenic-ring was obtained, where $\mathrm{C}_{9}$-cumulenic-ring was not initially flat after geometry optimization was applied, and consequently with partial aromaticity. In this case, our $\mathrm{C}_{17}$-cumulenic-ring is flat increasing in this way its aromaticity. This might be one reason to obtain an increased perpendicularity between chitosan copolymer unit and $\mathrm{C}_{17}$-cumulenic-ring. Another reason might be its degeneracy due to Jahn-Teller effect [18], because high degeneracy indicates high symmetry of the molecule, consequently the system tends to be distorted, in such way that when moving, the occupied levels are down and the unoccupied ones are up [26].

Our result of a nanocomposite formed with chitosan copolymer T-shape with $\mathrm{C}_{17}$-cumulenic-ring agree with the results presented by Chigo et al. in a study of the interaction among graphene-chitosan for a relaxed system doped with boron, in which they consider the interaction of pristine graphene with the monomer of chitosan (G + MCh: $\mathrm{C}_{6} \mathrm{H}_{13} \mathrm{O}_{5} \mathrm{~N}$ ) in different configurations, whereas we consider relaxed chitosan copolymer $\mathrm{C}_{14} \mathrm{H}_{24} \mathrm{~N}_{2} \mathrm{O}_{9}$ with $\mathrm{C}_{17}$ cumulenic-ring. While Chigo et al. found a perpendicular chitosan molecule linked to a carbon nanotube system, we obtained a $\mathrm{C}_{17}$ cumulenic-carbon ring perpendicularly linked to a chitosan copolymer system.

\section{Conclusions}

It is found one mechanism to figure out an optimized big molecular complex system by using DFT geometry optimization. The chitosan copolymer unit and the monocycle carbon-ring were relaxed first individually and second together in order to get stable systems. The latter has been suitable to optimize chitosan by adding activated carbon, in at least two geometry orientations, and it is observed the change in pore size distribution through the distance where the peaks of each radial distribution function calculated are located. It is predicted a nanocomposite constituted of an increase of carbon on chitosan at least in several ways. One is by modifying the whole structure of each reactant, which leads to a very stable new material useful to clean polluted water as an example. Another is that one unit of chitosan copolymer almost in T-shape with $\mathrm{C}_{17}$-cumulenic-ring, agrees with previous results reported in literature. This provides a new stable material which keeps the original structure of each reactant almost invariant, and it can be considered as nanocomposite $\mathrm{AC}+\mathrm{Ch}$ molecular complex system that can be applied in medicine as mitigating intoxication. The change in surface area allows increasing in toxic gas adsorption on this new material, or adsorption of another toxic substance inside of the body of a human being.

\section{Funding Acknowledgement}

This work was supported by Tecnológico Nacional de México [grant number TOL-PYR-2016-078].

\section{References}

1. Rinaudo M (2006) Chitin and chitosan: properties and applications. Prog Polym Sci 31: 603-632.

2. Khang G (2012) Handbook of Intelligent Scaffold for Tissue Engineering and Regenerative Medicine. CRC Press, Taylor and Francis, Boca Ratón, FL. 
3. Cardona V (2012) (Spanish) Physicochemical and structural preparation and characterization of a chitosan-based conducting gel. BSc Degree, Universidad del Valle, Santiago de Cali, Colombia.

4. Wang CM, Chang KS, Chung TW, Wu H (2004) Adsorption equilibria of aromatic compounds on activated carbon, silica gel, and $13 x$ zeolite. J Chem Eng Data 49: 527-531.

5. Lozano D, Cazorla D, Linares A (2002) Powdered Activated Carbons and Activated Carbon Fibers for Methane Storage: A Comparative Study. Energy \& Fuels 16: 1321-1328.

6. Smisek M, Cerny S (1970) Active carbon: manufacture, properties and applications. Elsevier, New York.

7. Wakabayashi T, Krätschmer W (2006) Carbon chain molecules in cryogenic matrices. In: Cataldo F (ed) Polyynes: Synthesis, Properties, and Applications. CRC Taylor and Francis: Boca Raton, FL, pp: 99-125.

8. Zavitsanos PD, Carlson GA (1973) Experimental study of the sublimation of graphite at high temperatures J Chem Phys 59: 2966

9. Cataldo F (ed) (2006) Polyynes: Synthesis, Properties, and Applications. CRC Taylor and Francis: Boca Raton, FL.

10. Torelli T, Mitas L (2000) Electron Correlation in C4N+2 Carbon Rings: Aromatic Versus Dimerized Structures. Phys Rev Lett 85: 1702-1705.

11. Remya K, Suresh CH (2016) Carbon rings: Study of geometry, aromaticity, intermolecular carbon-carbon interactions and stability. RSC Adv 6(50): 44261-44271.

12. Baughmann RH (2006) Dangerously Seeking Linear Carbon. Science 312: 1009-1110.

13. Ding H, Schmidt TW, Pino T, Güthe F, Maier JP (2003) Towards bulk behavior of long hydrogenated carbon chains? Phys Chem Chem Phys 5 4772 .

14. Cataldo F, Keheyan Y (2006) Polyynes: Simple synthesis in solution through the glaser reaction. In: Cataldo F (ed) Polyynes: Synthesis, Properties, and Applications. CRC Taylor and Francis: Boca Raton, FL, pp: 493498

15. Liu M, Artyukhov VI, Lee H, Xu F, Yakobson BI (2013) Carbyne from First Principles: Chain of C Atoms, a Nanorod or a Nanorope. ACS Nano,

16. Bylaska EJ, Weare JH, Kawai R (1998) Development of Bond-Length Alternation in Very Large Carbon Rings: LDA Pseudopotential Results. Phys Rev B 58: R7488-R7491.

17. Saito M, Okamoto Y (1999) Second Order Jahn-Teller Effect on Carbon 4N+2 Member Ring Clusters. Phys. Rev. B 60: 8939-8942.

18. Jahn HA, Teller E (1937) Stability of Polyatomic Molecules in Degenerate Electronic States. I. Orbital Degeneracy. Proc R Soc London A 161: 220235.

19. Rubin Y, Kahr M et al (1991) The higher oxides of carbon C8nO2n (n = 3-5): synthesis, characterization, and x-ray crystal structure. Formation of cyclo[n] carbon ions $\mathrm{Cn}+(\mathrm{n}=18,24)$, $\mathrm{Cn}-(\mathrm{n}=18,24,30)$, and higher carbon ions including $\mathrm{C} 60+$ in laser desorption Fourier transform mass spectrometric experiments. J Am Chem Soc 113: 495.

20. Handschuh H, Ganteför G, Kessler B, bechthold PS, Eberhardt W (1995) Stable Configurations of Carbon Clusters: Chains, Rings, and Fullerenes. Phys Rev Lett 74: 1095-1098.

21. Wakabayashi T et al (1997) Photoelectron spectroscopy of Cn produced from laser ablated dehydroannulene derivatives having carbon ring size of $\mathrm{n}=12,16,18,20$, and $24 \mathrm{~J}$ Chem Phys 107: 4783.

22. Martin JML, El-Yazal J, François JP (1995) Structure and vibrational spectra of carbon clusters $\mathrm{C} n(\mathrm{n}=2-10,12,14,16,18)$ using density func- tional theory including exact exchange contributions. Chem Phys Lett 242: 570-579.

23. Atkins P, Friedman R (2005) Molecular Quantum Mechanics. 4th edn. Oxford University Press, New York.

24. Fukui K, Yonezawa T, Nagata C, Shingu H (1954) Molecular orbital theory of orientation in aromatic, heteroaromatic, and other conjugated molecules. J Chem Phys 22: 1433-1442.

25. Lewis G (1923) Valence and the Structure of Atoms and Molecule. The Chemical Catalog Company, inc.

26. Chigo E, Torres A, Hernández H (2014) Studies of graphene-chitosan interactions and analysis of the bioadsorption of glucose and cholesterol. Appl Nanosci 4: 911-918.

27. Kadokawa JI (2014) Preparation of Chitin-Based Nano-Fibrous and Composite Materials Using Ionic Liquids. In: Han-Chan C, Hua-Chia C, Thomas S (ed) Physical Chemistry of Macromolecules: Macro to Nanoscales, CRC Press, Apple Academic Press, New Jersey, pp 367-384

28. Rodriguez A, Chigo E, Hernandez H, Flores A (2013) Adsorption of chitosan on BN nanotubes: A DFT investigation. Appl Surf Sci 268: 259264.

29. Delley B (1995) DMol, a standard tool for density functional calculations; review and advances. In: Seminario JM, Politzer P (eds) Modern density functional theory: a tool for chemistry. Elsevier, Amsterdam, pp: 221-254.

30. Auckenthaler T, Blum V, Bungartz HJ, Huckle T, Johanni R, et al. (2011) Parallel solution of partial symmetric eigenvalue problems from electronic structure calculations. Parallel Computing 37: 783.

31. Delley B (1990) An all-electron numerical method for solving the local density functional for polyatomic molecules. J Chem Phys 92: 508.

32. Delley B (2000) From molecules to solids with the DMol3 approach. J Chem Phys 113: 7756-7764.

33. Perdew JP, Wang Y (1986) Accurate and simple density functional for the electronic xchange energy: Generalized gradient approximation. Phys Rev B 33: 8800-8802.

34. Perdew JP (1991) Generalized gradient approximations for exchange and correlation: A look backward and forward. Physica B 172:1-6.

35. Perdew JP, Wang Y (1992) Accurate and simple analytic representation of the electron-gas correlation energy. Phys Rev B 45:13244-13249.

36. Estrada E (2002) Physicochemical Interpretation of Molecular Connectivity Indices. J Phys Chem A 106: 9085-9091.

37. Pacheco JH, Zaragoza IP, Bravo A (2017) Interaction of small carbon molecules and zinc dichloride: DFT study. Rev Mex Fís 63: 97-110.

38. Atkins P, De Paula J (2010) Physical Chemistry. Freeman and Company, New York

39. Guibal E, Milot C, Tobin J (1998) Metal-anion sorption by chitosan beads: equilibrium and kinetic studies. Ind Eng Chem Res 37:1454-1463.

40. Isidro FJ, Pacheco JH, Desales LA (2017) Hydrogen Storage on lithium decorated zeolite templated carbon, DFT study. International Journal of Hydrogen Energy.

41. Hernández-Benitez D, Pacheco-Sánchez JH (2018) Optimization of Chitosan+Activated Carbon Nanocomposite. DFT Srudy. Arc Org Inorg Chem Sci 3(5): 436 .

42. Sakurai K, Maegawa T, Takahashi T (2000) Glass transition temperature of chitosan and miscibility of chitosan/poly( $N$-vinyl pyrrolidone) blends. Polymer 41: 7051-7056. 